
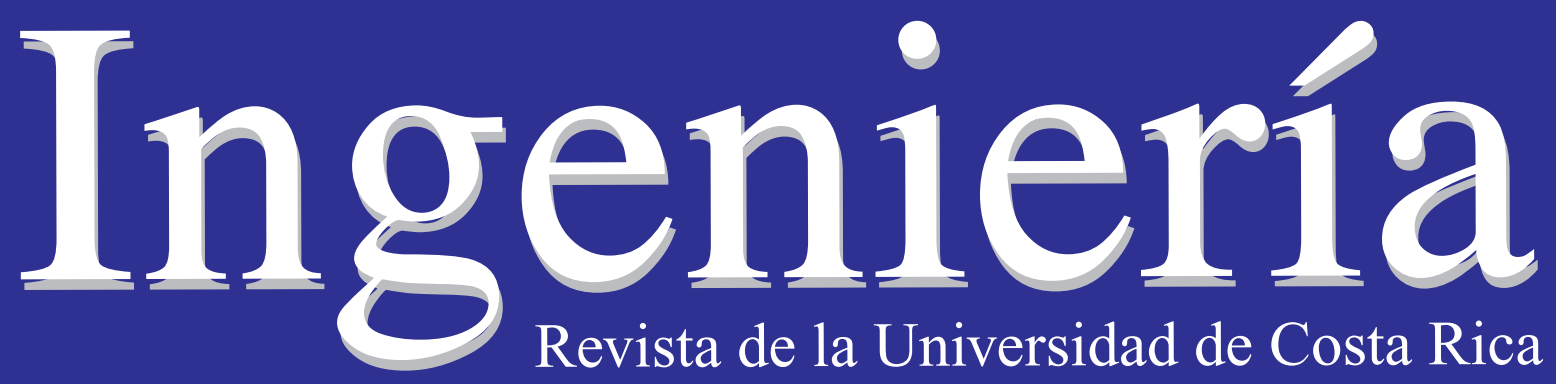

Revista de la Universidad de Costa Rica ENERO/ JULIO 2011 - VOLUMEN 21 Número (1)

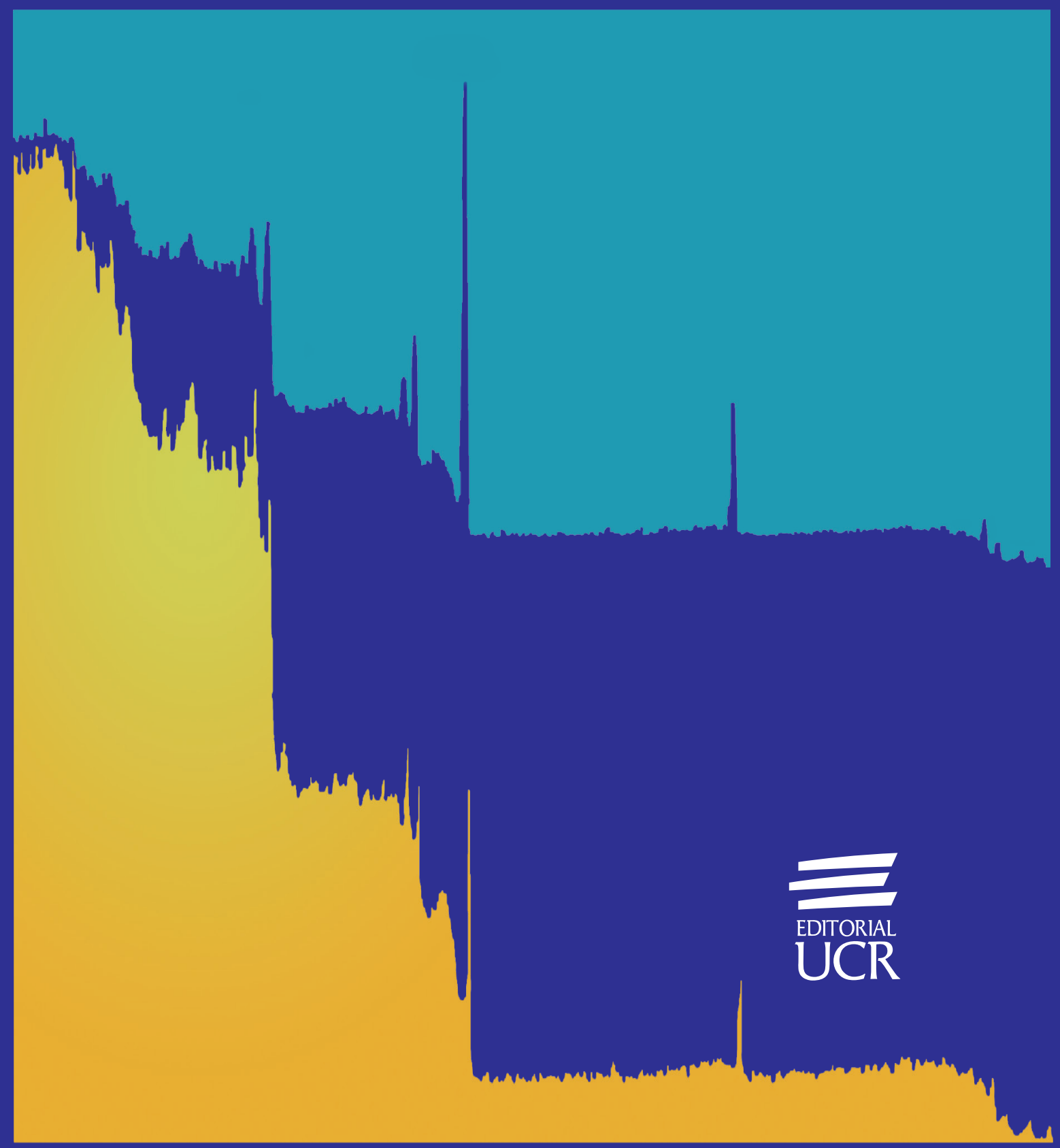




\title{
INCREMENTO DEL MARGEN DE CARGABILIDAD DEL SISTEMA ELÉCTRICO NACIONAL
}

\author{
Jairo Quirós Tortós
}

Eddie A. Araya Padilla

\begin{abstract}
Resumen
Esta publicación presenta la aplicación de una metodología para incrementar el margen de cargabilidad del sistema eléctrico nacional, bajo la restricción de un colapso de tensión de largo alcance. El método empleado utiliza el flujo de potencia convencional y el flujo óptimo de potencia para determinar la localización adecuada de acciones correctivas, como nuevos equipos de compensación, el despacho de carga y redespacho de generación. Es considerablemente factible que los resultados reflejen un incremento en el margen de cargabilidad del sistema cuando el análisis se lleva a cabo con el flujo óptimo de potencia; en comparación con un flujo de potencia convencional.
\end{abstract}

Palabras clave: Margen de cargabilidad, colapso de tensión, flujo de potencia, flujo óptimo de potencia..

\begin{abstract}
This paper presents the application of a methodology to improve the power system loading margins, in the costa rican electrical power system, constrained by long term voltage instability. The used method employs both conventional and optimal power flow to determine the adequate placement of corrective actions such as installation of new shunt device compensation, load shedding and unit commitment. It is considerably feasible that the results present an improvement in the loading margins of the power system using the optimal power flow method when compared to conventional power flow analysis..
\end{abstract}

Keywords: Loading margin, voltage collapse, load flow, optimal load flow.

Recibido: 09 de Febrero del 2010 • Aprobado: 31 de Marzo del 2011

\section{INTRODUCCIÓN}

El Sistema Eléctrico Nacional (SEN) está constituido por centros de generación localizados lejos de los centros de consumo. La mayor parte de generación de energía eléctrica en Costa Rica se encuentra en la zona norte del país, y la principal carga eléctrica se localiza en la gran área metropolitana.

El SEN, mostrado en la Figura 1, exhibe una estructura longitudinal conformada por líneas de transmisión de 230 y $138 \mathrm{kV}$. Este sistema, durante condiciones de máxima demanda o cuando ocurren contingencias, está propenso a un colapso de tensión ya que dicha problemática puede asociarse con la estructura del sistema de transmisión (CIGRE, 1992).
Debido a la topología relativamente débil del SEN, el 6 de marzo del 2001 a las 18:42 horas, el doble circuito Barranca - La Caja salió de servicio provocando que la línea Arenal - Ciudad Quesada - Toro - San Miguel pasara a operar con un nivel de carga mayor al permitido. En ese momento, el sistema carecía de un respaldo adecuado para mantener la tensión en niveles normales de operación, y su magnitud en las barras de distribución disminuyó. Ante esta situación, los transformadores regulables bajo carga entraron en operación, lo que provocó un flujo de potencia reactiva mayor de la red de transmisión hacia la red de distribución, ocasionando una disminución de tensión adicional en la red de transmisión. Como consecuencia del fenómeno a los 4 minutos de iniciado el evento se originó un 
colapso de tensión en todo el territorio nacional (ICE, 2001).

A partir de un análisis post-mortem de dicho evento y con base en experiencias reales de otros sistemas de potencia (Taylor, 1994), se concluye que el fenómeno se considera de largo alcance desde el punto de vista temporal. Además, se hace evidente la incapacidad del sistema para suministrar potencia reactiva en las barras críticas de la red. Dicha situación, sugiere una estrecha relación entre la inestabilidad de tensión y la localización y reserva de potencia reactiva (Tiranuchit et al., 1988).

Este problema cuasi estacionario se reconoce en la literatura técnica (Taylor, 1994) como inestabilidad de tensión de largo alcance, el cual es posible evitar si el operador del sistema dispone de medidas preventivas adecuadas.

Entre las medidas preventivas se destaca en primera instancia la localización de aquellos nodos eléctricamente débiles del sistema con la finalidad de sugerir algún tipo de compensación de potencia reactiva, o en su defecto, durante el desarrollo de la caída de tensión, el despacho de carga en una forma sistemática.

En vista de lo anterior, como un primer paso para identificar los nodos débiles del sistema, se ejecuta un flujo de potencia convencional para establecer un punto operativo del sistema. Con esta información y con la finalidad de disminuir las pérdidas de potencia reactiva del sistema se ejecuta un flujo óptimo de potencia, para

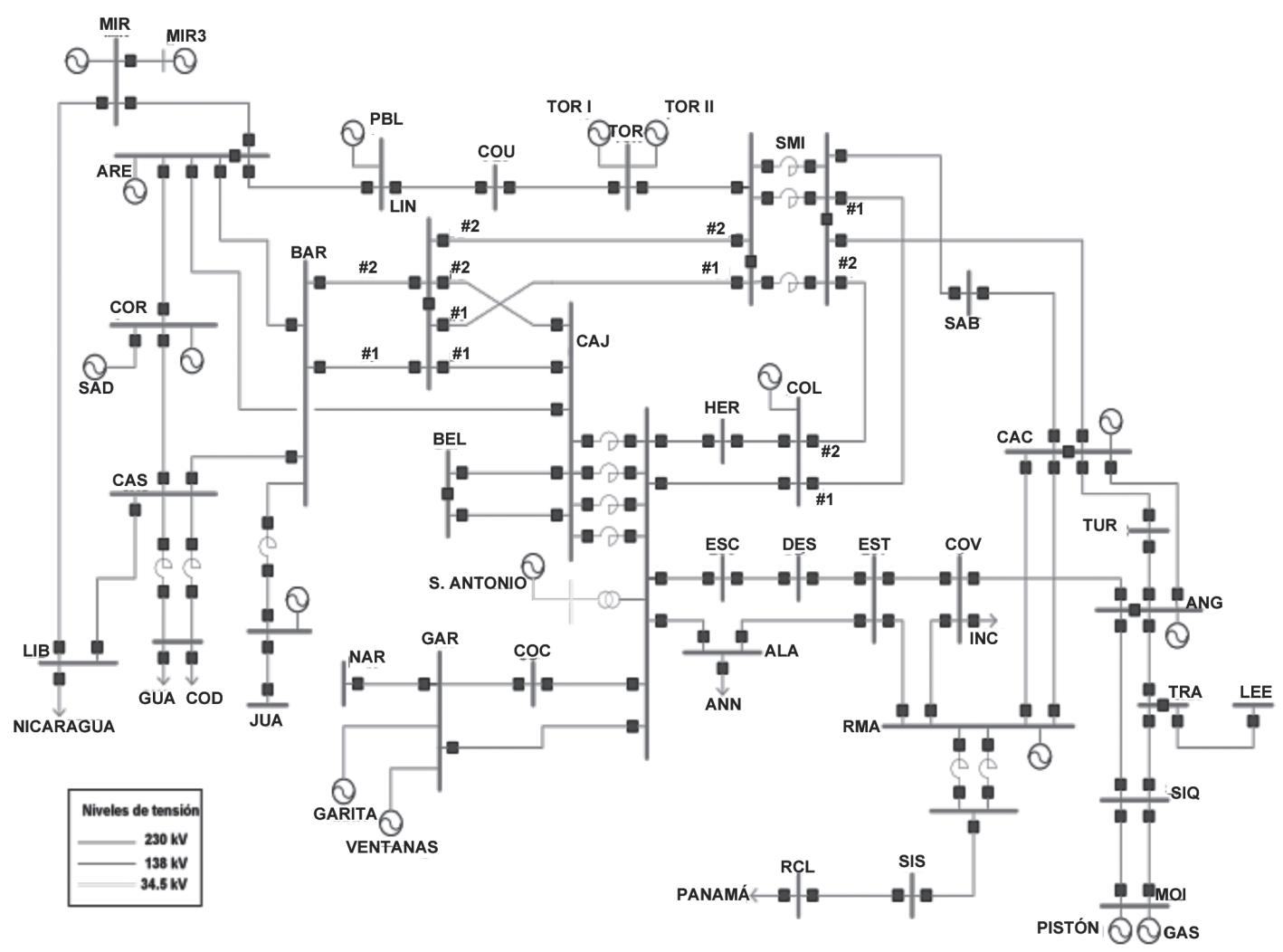

Figura 1. Diagrama unifilar del SEN.

Fuente: (Quirós, 2009) 


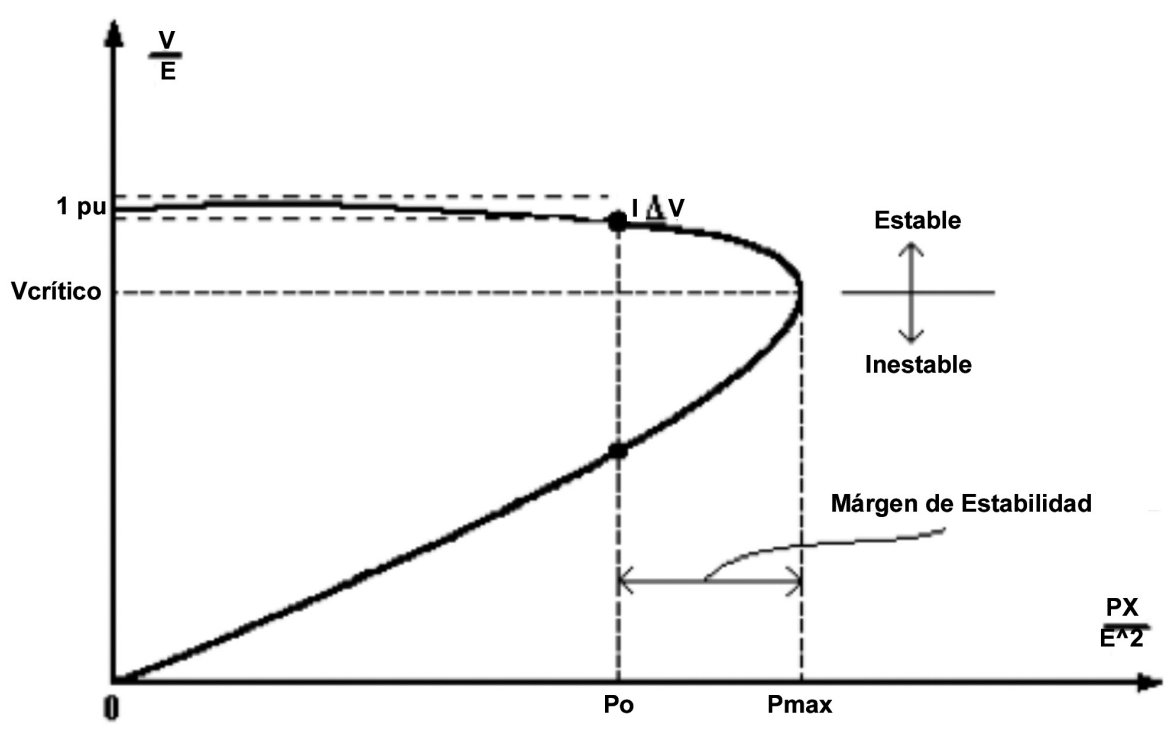

Figura 2. Forma típica de una curva P-V normalizada.

Fuente: (Quirós, 2009)

valorar el incremento obtenido en el margen de cargabilidad, con restricciones de un colapso de tensión. Este incremento del margen de cargabilidad es el objetivo de estudio de esta publicación.

\section{INDICES PARA DETERMINAR EL MARGEN DE ESTABILIDAD DE TENSIÓN}

Para determinar el límite estático de tensión, se utilizan indicadores de cercanía al punto de colapso. Dichos indicadores se denominan "índices" cuando son parámetros matemáticos sin una clara interpretación física (por ejemplo, el determinante de un Jacobiano), y "márgenes" si son una magnitud física (por ejemplo, cantidad de potencia activa) (Verayiah \& Zainal, 2008). Estos indicadores generalmente están relacionados con la cargabilidad del sistema, entre lo que se destacan las curvas P-V y V-Q.

\subsection{Curvas P-V, índices y márgenes derivados}

Las curvas P-V son utilizadas para el análisis conceptual de la estabilidad de tensión. Permiten determinar la máxima potencia activa que se puede transmitir hacia un centro de carga, sin producir colapso de tensión (Taylor, 1994). Sin embargo, en un ambiente multimáquina es necesario destacar que $\mathrm{P}$ es la carga total en un área y que $\mathrm{V}$ es la tensión en una barra crítica o representativa.

Estas curvas, muestran la forma en que disminuye el nivel de tensión en una barra conforme aumenta la transferencia de potencia activa. Este comportamiento se muestra en la Figura 2, donde se observa que conforme aumenta la potencia activa la tensión empieza a disminuir hasta alcanzar un punto crítico (p.c), donde la potencia activa entregada es máxima.

Este punto crítico representa la condición para la cual ocurre el colapso de tensión y depende de la impedancia de la red, la tensión y 
del factor de potencia de la carga. Para factores de potencia en atraso o unitario, el comportamiento de estas curvas es predecible. Sin embargo, para cargas con factor de potencia en adelanto, el comportamiento de la curva no resulta tan obvio (Van Cutsem \& Vournas, 1998).

\subsection{Curvas V-Q, índices y márgenes derivados}

Las curvas V-Q permiten determinar la reserva de potencia reactiva en una barra y el nivel de tensión en que ocurrirá el colapso de tensión en la misma. Estas curvas pueden obtenerse a partir de los resultados del flujo de potencia (Van Cutsem \& Vournas, 1998) y representan los márgenes de estabilidad de tensión.

La Figura 3 muestra la variación de la potencia reactiva en función de la tensión, manteniendo el factor de potencia y el valor de la potencia activa constantes. Además, se indica la región de estabilidad y el margen de potencia reactiva de la barra.

Dentro de las ventajas de las curvas V-Q se destacan (Taylor, 1994):

- La magnitud de tensión está estrechamente relacionada con la potencia reactiva, y por tanto, las curvas V-Q proveen el margen de potencia reactiva en la barra de estudio.

- La curva V-Q puede ser calculada en los puntos de operación de la curva P-V para pruebas de robustez del sistema.

- La pendiente de la curva V-Q indica la rigidez de la barra bajo análisis.

- El margen de potencia reactiva del punto de operación, puede ser obtenido directamente.

Dentro de las desventajas de las curvas V-Q, se destacan (Taylor, 1994):

- Para diferentes contingencias y transferencia de potencia aparente es necesario la construcción de muchas curvas V-Q en diferentes barras.

- Las curvas V-Q solamente indican la compensación de potencia reactiva necesaria en una barra, no la compensación del sistema.

\section{FLUJO DE POTENCIA CONVENCIONAL Y FLUJO DE POTENCIA ÓPTIMO}

Dos métodos para determinar el flujo de potencia en el sistema de potencia serán

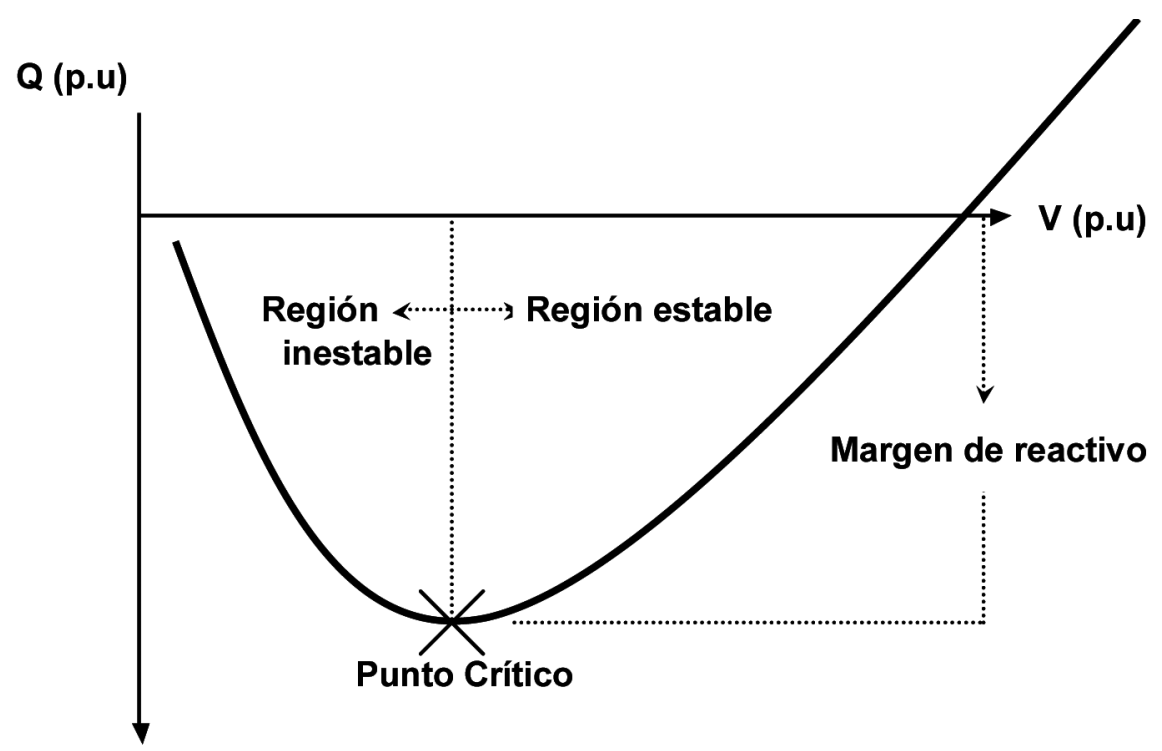

Figura 3. Forma típica de una curva V-Q normalizada.

Fuente: (Quirós, 2009). 
utilizados. El primero, flujo de potencia convencional ha sido utilizado por el ICE hasta el momento. Mientras que el segundo, flujo de potencia óptimo, será propuesto en este artículo.

\subsection{Flujo de potencia convencional}

Diferentes métodos para resolver las ecuaciones no lineales que modelan el comportamiento del sistema de potencia han sido desarrollados durante las últimas décadas (Kundur, 1994). Dicho comportamiento puede ser representado de acuerdo a la Ecuación 1.

$$
\left[\begin{array}{c}
\tilde{I}_{1} \\
\tilde{I}_{2} \\
\ldots \\
\tilde{I}_{n}
\end{array}\right]=\left[\begin{array}{cccc}
Y_{11} & Y_{12} & \ldots & Y_{1 n} \\
Y_{21} & Y_{22} & \ldots & Y_{2 n} \\
\ldots & \ldots & \ldots & \ldots \\
Y_{n 1} & Y_{n 2} & \ldots & Y_{n n}
\end{array}\right]\left[\begin{array}{c}
\tilde{V}_{1} \\
\tilde{V}_{2} \\
\ldots \\
\tilde{V}_{n}
\end{array}\right]
$$

Donde:

- $\mathrm{n}$ es el número total de barras del sistema,

- $\mathrm{Y}_{i i}$ es la admitancia propia de la barra $i$,

- $\quad \mathrm{Y}_{i j}$ es la admitancia mutua entre la barra $i$ y $j$

$\tilde{V}_{i}$ es el fasor de la tensión a tierra en la barra $i \mathrm{e}$

- $\quad \tilde{I}_{i}$ es el fasor de la corriente eléctrica fluyendo a la barra $i$.

Para obtener la solución de la ecuación (1), métodos como el Gauss-Seidel, NewtonRaphson y derivaciones de este último han sido desarrollados(Kundur, 1994). Este articulo hará uso del Newton-Raphson completo, el cual hace uso de las ecuaciones de potencia representadas en (3) y (4).

$$
\tilde{S}_{k}=P_{k}+j Q_{k}=\tilde{V}_{k} \tilde{I}_{k}^{*}
$$

$$
P_{k}=V_{k} \sum_{m=1}^{n}\left(G_{k m} V_{m} \cos \theta_{k m}+B_{k m} V_{m} \sin \theta_{k m}\right)
$$

donde $\tilde{I}_{k}=\sum_{m=1}^{n} \tilde{Y}_{k m} \tilde{V}_{m}$ de acuerdo a (1).

$Q_{k}=V_{k} \sum^{n}\left(G_{k m} V_{m} \sin \theta_{k m}-B_{k m} V_{m} \cos \theta_{k m}\right)$

Considerando las ecuaciones anteriores, la formulación matemática para resolver flujos de potencia utilizando la formulación de NewtonRaphson es presentada en (5).

$$
\left[\begin{array}{c}
\Delta \mathbf{P} \\
\Delta \mathbf{Q}
\end{array}\right]=\left[\begin{array}{ll}
\frac{\partial \mathbf{P}}{\partial \boldsymbol{\theta}} & \frac{\partial \mathbf{P}}{\partial \mathbf{V}} \\
\frac{\partial \mathbf{Q}}{\partial \boldsymbol{\theta}} & \frac{\partial \mathbf{Q}}{\partial \mathbf{V}}
\end{array}\right]\left[\begin{array}{c}
\Delta \boldsymbol{\theta} \\
\Delta \mathbf{V}
\end{array}\right]
$$

\subsection{Flujo de potencia óptimo}

Los flujos de potencia óptimo se basan en la solución óptima del problema de flujos de potencia. Se trata de un método no lineal que resuelve la ecuación presentada en (6).

$$
\min _{\mathbf{x}} f(\mathbf{x})
$$

sujeto a

$$
\begin{aligned}
& g(\mathbf{x})=0 \\
& h(\mathbf{x}) \leq 0 \\
& \mathbf{x}_{\min } \leq \mathbf{x} \leq \mathbf{x}_{\max }
\end{aligned}
$$

Donde el vector $\mathbf{x}$ es la variable a optimizar, $f(\mathbf{x})$ es la función objetivo, $g(\mathbf{x})$ son las restricciones con igualdad, $h(\mathbf{x})$ son las restricciones desiguales, $\mathbf{x}_{\text {min }}$ and $\mathbf{x}_{\text {max }}$ representan el mínimo y máximo valor de los parámetros, respectivamente. Las funciones $f(\mathbf{x}), g(\mathrm{x})$ and $h(\mathbf{x})$ se asumen continuas y diferenciables al menos dos veces para $\mathbf{x} \in \mathbb{R}^{n_{\mathbf{x}}}$ En la formulación del flujo de potencia óptimo, 
el vector $\mathrm{x}$ está constituido por $\mathrm{n}_{b} \mathrm{x} 1$ vectores de fase de la tensión y su magnitud y de $\mathrm{n}_{g} \times 1$ vectores de potencia activa generada y potencia reactiva generada.

$$
\mathbf{x}=\left[\begin{array}{llll}
\theta & V_{m} & P_{g} & Q_{g}
\end{array}\right]
$$

\subsection{Principales diferencias en los métodos}

Ambos métodos utilizados en este artículo han sido ampliamente estudiados en el campo de los sistemas de potencia. Las diferencias de un método a otro se basan en la solución óptima obtenida haciendo uso de la herramienta OPF. La metodología propuesta en la Sección 3.1 resuelve la Ecuación 1 sin consideraciones físicas que deben ser analizadas en la operación básica del sistema de potencia.

El uso de la herramienta de flujos de potencia óptimo tiene como principal característica que resuelve la Ecuación 1, manteniendo todas las variables eléctricas dentro de los límites de operación permisibles, así como que busca la solución optima para dichas variables. En este artículo, dicha herramienta se utilizará para reducir las pérdidas de potencia reactiva en la red de transmisión, problema relacionado con la estabilidad de tensión.

\section{BARRAS DÉBILES DEL SISTEMA ELÉCTRICO NACIONAL}

Para determinar las barras débiles del SEN, se usa como criterio de análisis, la estabilidad de tensión del sistema. A partir de esta información, se determina el punto operativo de colapso del sistema, con lo cual se cuantifica el margen de cargabilidad del mismo.

\subsection{Sistema Eléctrico Nacional y casos base}

Los casos bases consideradas para este estudio se detallan en el Cuadro 1. Dicha información fue suministrada por el ICE (Quirós, 2009) y corresponden a estimaciones para el año 2010.

A partir de los casos base, se realizan simulaciones en régimen permanente y se obtienen los flujos de potencia, tanto con la herramienta del CPF y del OPF. Con el OPF se minimizan las pérdidas de potencia reactiva, problema relacionado con la estabilidad de tensión.

Una vez realizadas las comparaciones en régimen permanente, de acuerdo a los puntos de operación definidos, se realizan cincuenta diferentes contingencias (Quirós, 2009), para determinar cuáles eventos son los más severos sobre la red.

$\mathrm{Al}$ simular estas contingencias en el SEN, operando con los despachos estimados inicialmente por la UEN - CENCE (Quirós, 2009), se determina que el Flujo de potencia Norte Centro (FNC) máximo estimado (500 MW para escenario de demanda máxima de verano y de $567 \mathrm{MW}$ para el escenario de demanda máxima de invierno), no provoca colapso de tensión alguno, sobre la topología de la red considerada para el año 2010. Debido a lo anterior, se debe

Cuadro 1. Casos base para el año 2010.

Escenario de la demanda

Verano (18:30)

Invierno (18:30)
Demanda máxima de potencia (MW) 
Cuadro 2. Barras críticas consideradas en el análisis.

\begin{tabular}{|c|c|}
\hline Nombre de la barra & Nivel de tensión $(\mathrm{kV})$ \\
\hline Barranca & 230 \\
\hline Garabito & 230 \\
\hline La Caja & 230 \\
\hline La Caja & 138 \\
\hline Lindora & 230 \\
\hline San Miguel & 230 \\
\hline San Miguel & 138 \\
\hline Escazú & 138 \\
\hline Desamparados & 138 \\
\hline Alajuelita & 138 \\
\hline El Este & 138 \\
\hline Heredia & 138 \\
\hline Colima & 138 \\
\hline Sabanilla & 138 \\
\hline
\end{tabular}

Fuente: (Quirós, 2009).

realizar un redespacho de generación para incrementar el FNC (790 MW y 774 MW para los escenarios de demanda máxima de verano e invierno, respectivamente), de modo tal, que el sistema presente problemas de estabilidad de tensión ante las contingencias definidas.

Una vez identificadas las contingencias, se consideraron como "barras críticas" aquellas donde se ha determinado que existen problemas de estabilidad de tensión y de reserva de potencia reactiva. La lista de las barras críticas consideradas para el análisis se muestra en el Cuadro 2.

\subsection{Punto operativo de colapso y margen de estabilidad de tensión}

En esta sección se procede a determinar el punto de colapso de tensión del SEN y se evalúa el margen de estabilidad de tensión, para diferentes condiciones de operación. Con esta información se lleva a cabo el análisis de estabilidad, para los dos escenarios considerados en el Cuadro 1.

\subsubsection{Demanda máxima de verano, 18:30 horas}

Para el caso base de "demanda máxima de verano 2010", se realizan simulaciones tanto con la herramienta del CPF, como con el OPF.

En el Cuadro 3 se presenta la potencia de la generación, el consumo de la carga y las pérdidas para el caso del CPF, mientras que en el Cuadro 4 se muestran los mismos datos, utilizando la herramienta OPF.

Al comparar los resultados indicados en los Cuadros 3 y 4, se observa como las pérdidas de potencia en el caso de la herramienta OPF fueron menores que las obtenidas en el caso del CPF. De esta forma, se obtiene una disminución del 9,47 $\%$, para las pérdidas de potencia activa; mientras que para la potencia reactiva, ésta disminuye en un $24,41 \%$.

Una vez realizadas las comparaciones, entre el CPF y el OPF, se observa que debido a la topología de la red considerada para el año 2010, y de acuerdo al FNC estimado de 500 $\mathrm{MW}$, no hay contingencia alguna que provoque inestabilidad de tensión en la red (Quirós, 2009). Debido a lo anterior, se realiza un redespacho para incrementar el FNC, con el objetivo de graficar las curvas $\mathrm{P}-\mathrm{V}$ y encontrar el punto del colapso de tensión en condiciones sin falla.

Ahora bien, para obtener el punto de colapso del sistema se determina que las contingencias más severas sobre la red de potencia son: la salida de la barra A de Arenal (ARENAL) y la salida de la línea Cariblanco - San Miguel (CU1). A partir de estas contingencias, se procede a obtener las curvas P-V y V-Q para cuantificar la cercanía al punto de colapso de tensión.

A continuación se muestran las curvas $\mathrm{P}-\mathrm{V}$ y V-Q para las barras consideradas en el análisis 


\section{La Caja 230}

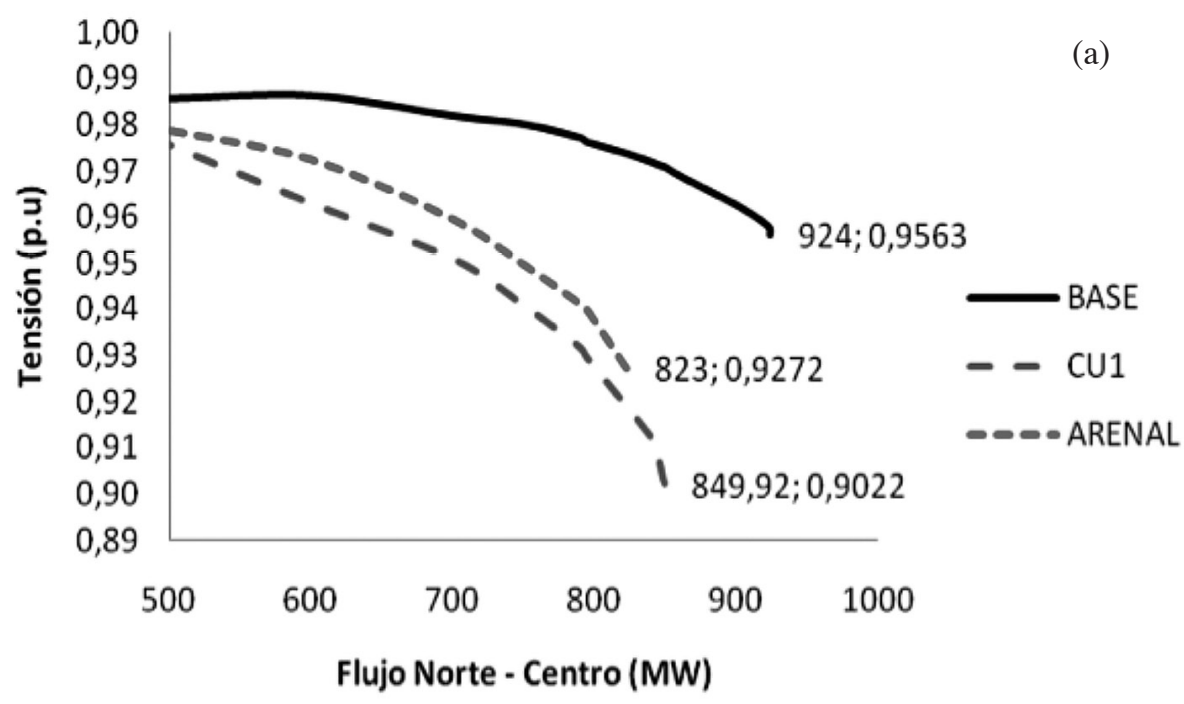

La Caja 230

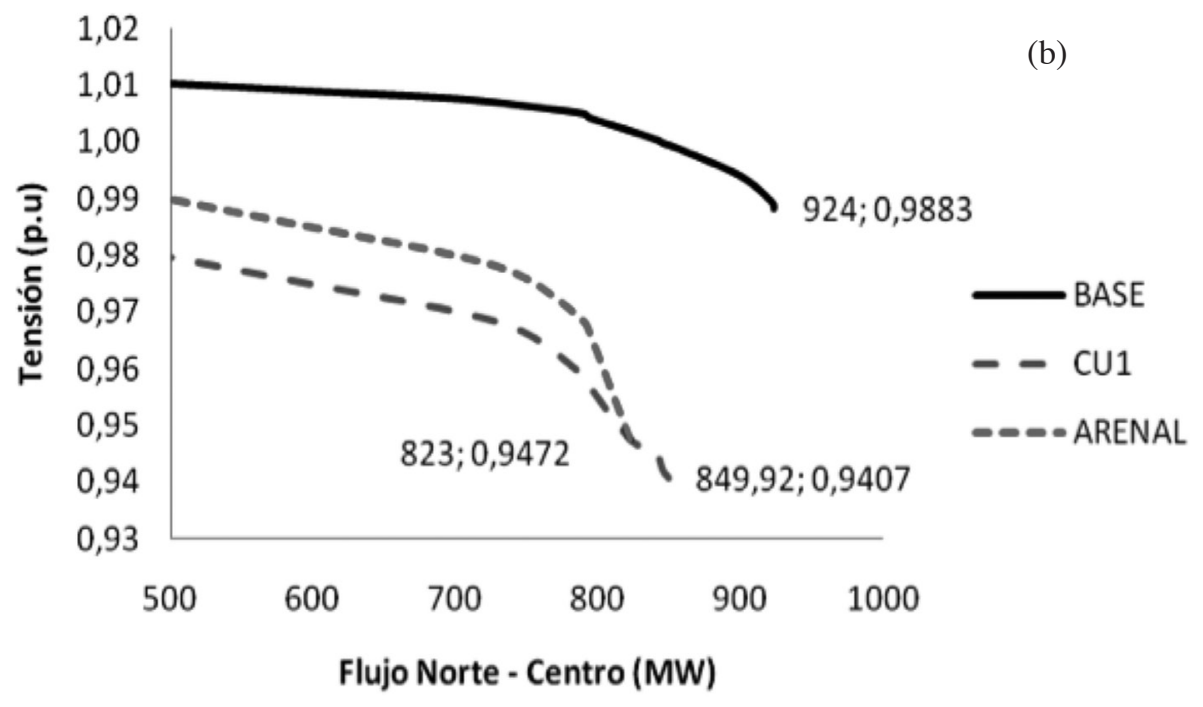

Figura 4. Curva P-V en barra La Caja 230 para caso base de verano máxima, considerando el FNC (a) CPF (b) OPF.

Fuente: (Quirós, 2009) 
La Caja 230

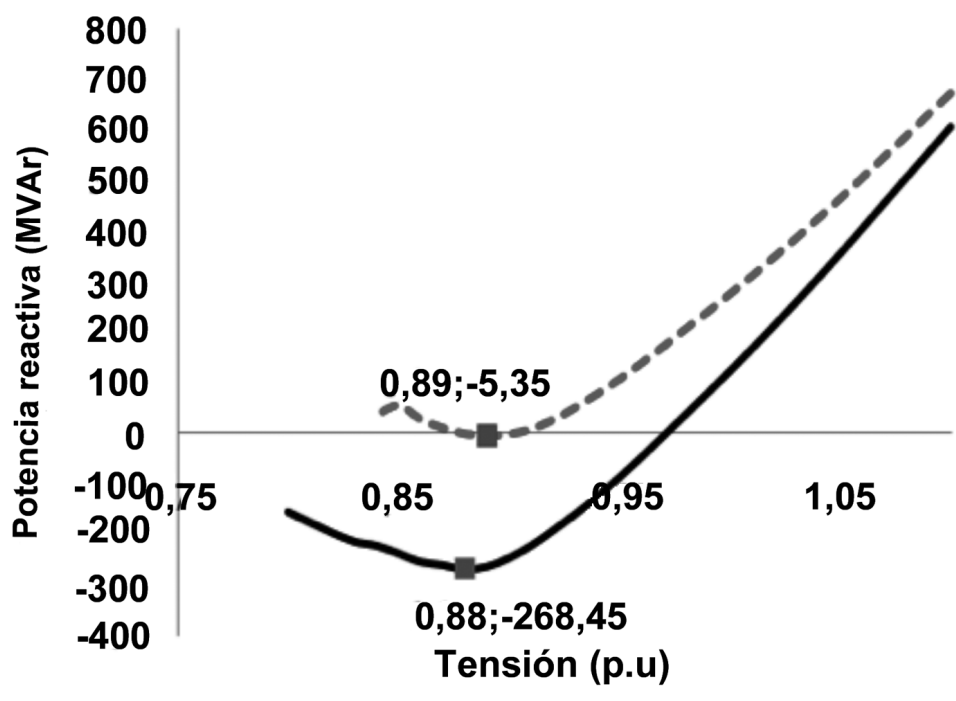

La Caja 230

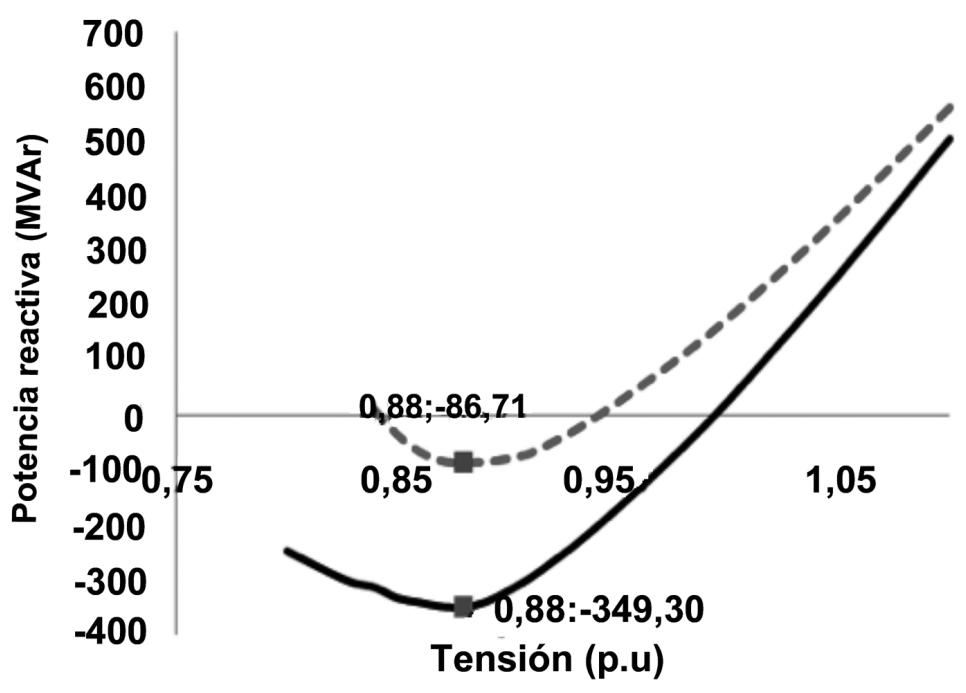

(a)

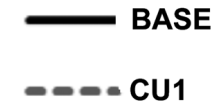

Base

$-\infty-$ CU1

Figura 5. Curva V-Q en La Caja 230 para caso base de demanda máxima de verano y la salida de la línea Cariblanco - San Miguel (a) CPF (b) OPF.

Fuente: (Quirós, 2009) 
Cuadro 3. Análisis de potencias, para el caso base, usando CPF.

$\begin{array}{lcc}\text { Item } & \text { MW } & \text { MVAr } \\ \text { Generación } & 1155,30 & 251,00 \\ \text { Carga } & 1129,00 & 369,00 \\ \text { Perdidas } & 26,40 & 380,60\end{array}$

Fuente: (Quirós, 2009).

Cuadro 4. Análisis de potencias, para el caso base, usando OPF.

$\begin{array}{lcc}\text { Item } & \text { MW } & \text { MVAr } \\ \text { Generación } & 1155,60 & -134,40 \\ \text { Carga } & 1129,00 & 369,00 \\ \text { Perdidas } & 23,90 & 287,70\end{array}$

Fuente: (Quirós, 2009).

de criticidad del sistema (Cuadro2), donde se compara la curva P-V en la barra de $230 \mathrm{kV}$ de la Subestación La Caja, tanto con la herramienta del CPF como con la del OPF.

En la Figura 4 se observan las curvas P-V del caso de demanda máxima de verano al considerar la solución con OPF, se determina que la tensión de colapso aumenta en aproximadamente 0,03 p.u.

Una vez obtenidas las curvas $\mathrm{P}-\mathrm{V}$, se puede observar que ambas contingencias tienen puntos de colapso de tensión diferente, por lo que se aclara, que las curvas V-Q, considerando la salida de la barra A de Arenal, se realizan para un FNC de 790 MW. Por otro lado, para realizar las mismas curvas, pero considerando la salida de la línea Cariblanco - San Miguel, el FNC despachado es de 848,86 MW.

En las Figuras 5 y 6 se detalla la curva V-Q para la barra de $230 \mathrm{kV}$ de La Caja, tanto para la salida de la línea Cariblanco - San Miguel, como para la salida de la barra A de Arenal, respectivamente.

Es importante observar la diferencia en realizar el análisis con el OPF y con el CPF. En la Figura 5, se detalla el margen de potencia reactiva de la barra de $230 \mathrm{kV}$ en la Subestación La Caja, el cual es de 5,35 MVAr ante la salida de la línea Cariblanco - San Miguel, utilizando el $\mathrm{CPF}$, mientras que para el mismo caso, utilizando el OPF, se obtiene un margen de potencia reactiva de 86,71 MVAr.

En el caso de la salida de la barra A de Arenal (Figura 6), el margen de potencia reactiva en la barra de $230 \mathrm{kV}$ en la Subestación La Caja, utilizando el CPF es de 67,38 MVAr y 148,45 MVAr utilizando el OPF. Al igual que en la contingencia anterior, se observa como el margen de potencia reactiva en las barras del sistema aumenta significativamente utilizando el OPF.

En el Cuadro 5 se muestra con mayor detalle el punto de colapso del sistema cuando se origina la salida de la línea Cariblanco - San Miguel, o en su defecto, cuando ocurre la salida de la barra A de Arenal.

Por otro lado, es importante notar en el Cuadro 5, que en algunas barras analizadas, la tensión aumenta hasta en 0,0436 p.u (barra de Alajuelita para la falla de la línea Cariblanco San Miguel).

Una vez disponibles las curvas P-V para el caso de demanda máxima de verano, se procede a elaborar el Cuadro 6, donde se detalla el margen de potencia reactiva para cada barra del Cuadro 2 .

En el Cuadro 6 se muestra un incremento en el margen de potencia reactiva de cada barra del sistema, siendo la salida de la barra A de Arenal, el caso que provoca la mayor inestabilidad del sistema. Lo anterior se refleja en la cantidad de potencia que se transfiere del norte hacia el centro y del margen de potencia reactiva en las barras de la red.

El Cuadro 6 muestra como, ante las contingencias consideradas, la red experimenta 


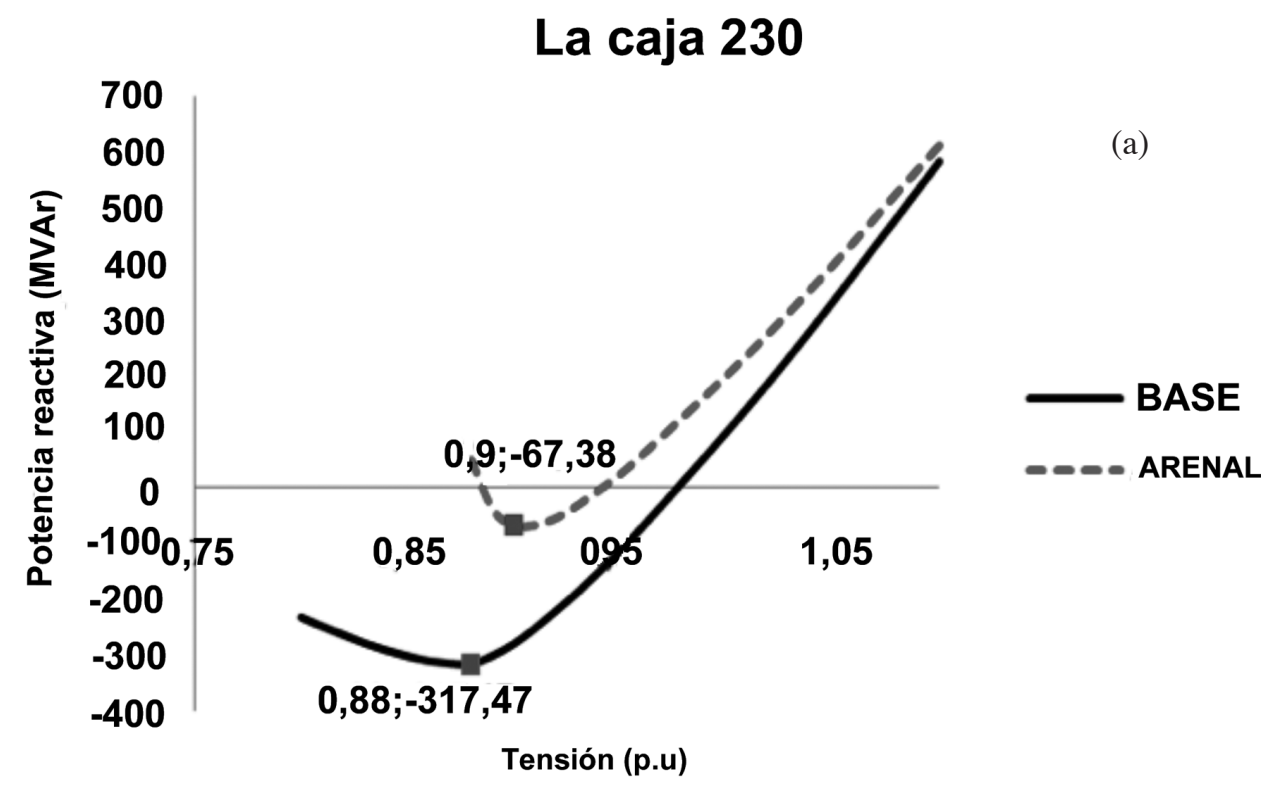

\section{La Caja 230}

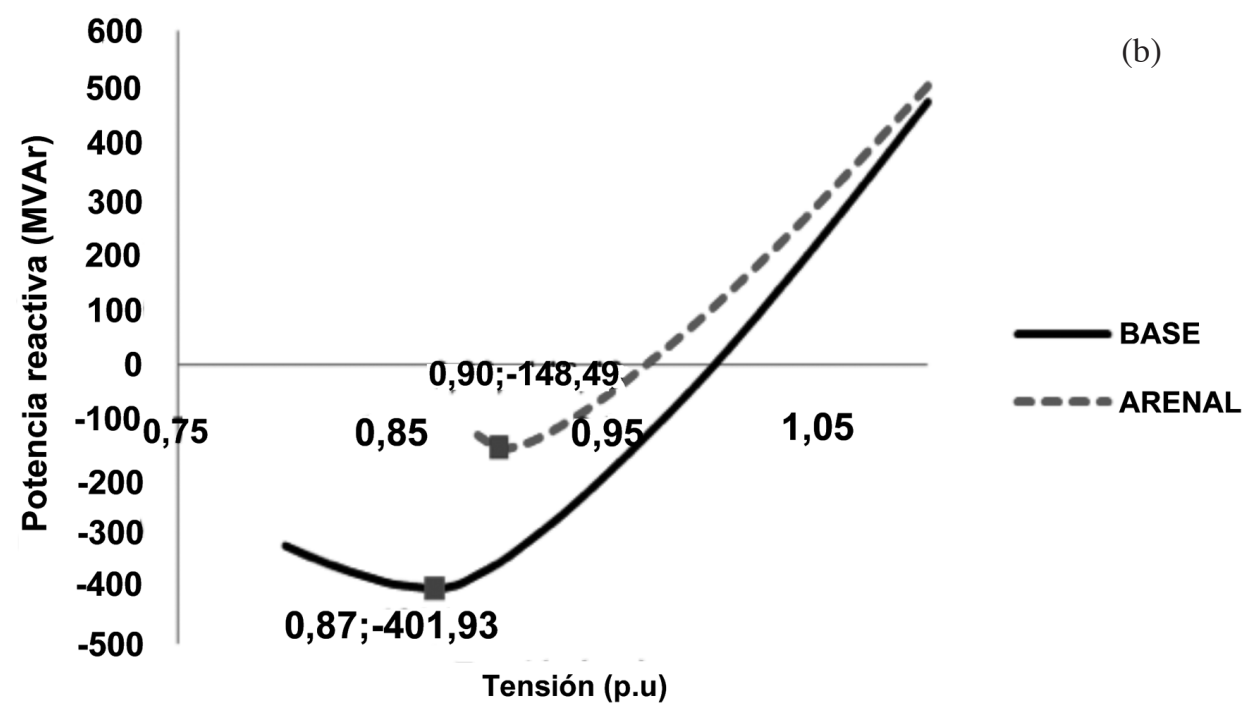

Figura 6. Curva V-Q en La Caja 230 para caso base de demanda máxima de verano y la salida de la barra A de Arenal (a) CPF (b) OPF.

Fuente: (Quirós, 2009) 
Cuadro 5. Puntos de colapso del SEN, obtenidos de las curvas P-V, en demanda máxima de verano.

\begin{tabular}{|c|c|c|c|c|c|c|c|c|}
\hline \multirow{3}{*}{$\begin{array}{l}\text { Nombre } \\
\text { de la barra }\end{array}$} & \multicolumn{4}{|c|}{ Falla línea Cariblanco - San Miguel } & \multicolumn{4}{|c|}{ Falla barra A de Arenal } \\
\hline & \multicolumn{2}{|c|}{$\mathrm{CPF}$} & \multicolumn{2}{|c|}{ OPF } & \multicolumn{2}{|c|}{ CPF } & \multicolumn{2}{|c|}{ OPF } \\
\hline & $\mathbf{P}(\mathbf{M W})$ & V (p.u) & $\mathbf{P}(\mathbf{M W})$ & $\mathbf{V}(\mathbf{p . u})$ & $\mathbf{P}(\mathbf{M W})$ & V (p.u) & $\mathbf{P}(\mathbf{M W})$ & V (p.u) \\
\hline Barranca & 849,92 & 0,9228 & 849,92 & 0,9553 & 823 & 0,9328 & 823 & 0,9528 \\
\hline Garabito & 849,92 & 0,9258 & 849,92 & 0,9577 & 823 & 0,9338 & 823 & 0,9538 \\
\hline La Caja & 849,92 & 0,9022 & 849,92 & 0,9407 & 823 & 0,9272 & 823 & 0,9472 \\
\hline Lindora & 849,92 & 0,9039 & 849,92 & 0,9419 & 823 & 0,9291 & 823 & 0,9491 \\
\hline San Miguel & 849,92 & 0,9085 & 849,92 & 0,9461 & 823 & 0,9358 & 823 & 0,9558 \\
\hline Escazú & 849,92 & 0,9330 & 849,92 & 0,9754 & 823 & 0,9553 & 823 & 0,9753 \\
\hline Desamparados & 849,92 & 0,9368 & 849,92 & 0,9779 & 823 & 0,9577 & 823 & 0,9777 \\
\hline Alajuelita & 849,92 & 0,9345 & 849,92 & 0,9781 & 823 & 0,9572 & 823 & 0,9772 \\
\hline El Este & 849,92 & 0,9485 & 849,92 & 0,9851 & 823 & 0,9700 & 823 & 0,9900 \\
\hline Heredia & 849,92 & 0,9294 & 849,92 & 0,9718 & 823 & 0,9536 & 823 & 0,9736 \\
\hline Colima & 849,92 & 0,9343 & 849,92 & 0,9754 & 823 & 0,9582 & 823 & 0,9782 \\
\hline Sabanilla & 849,92 & 0,9418 & 849,92 & 0,9783 & 823 & 0,9645 & 823 & 0,9845 \\
\hline
\end{tabular}


Cuadro 6. Margen de potencia reactiva, en demanda máxima de verano.

\begin{tabular}{|c|c|c|c|c|}
\hline \multirow[t]{2}{*}{ Nombre de la barra } & \multicolumn{2}{|c|}{$\begin{array}{c}\text { Falla línea Cariblanco - San Miguel } \\
\text { Margen de Q (MVAr) }\end{array}$} & \multicolumn{2}{|c|}{$\begin{array}{c}\text { Falla barra A de Arenal } \\
\text { Margen de Q (MVAr) }\end{array}$} \\
\hline & $\mathrm{CPF}$ & OPF & $\mathrm{CPF}$ & OPF \\
\hline Barranca & 5,21 & 79,64 & 57,60 & 122,01 \\
\hline Garabito & 5,07 & 80,05 & 57,05 & 121,17 \\
\hline La Caja 230 & 5,35 & 86,71 & 67,38 & 148,49 \\
\hline La Caja 138 & 5,90 & 92,84 & 75,29 & 162,38 \\
\hline Lindora & 5,71 & 86,04 & 67,88 & 147,84 \\
\hline San Miguel 230 & 4,92 & 92,33 & 70,74 & 155,53 \\
\hline San Migue 138 & 5,34 & 98,33 & 77,91 & 162,09 \\
\hline Escazú & 5,96 & 93,81 & 73,47 & 162,50 \\
\hline Desamparados & 7,63 & 100,03 & 80,23 & 176,89 \\
\hline Alajuelita & 6,68 & 96,21 & 76,26 & 168,10 \\
\hline El Este & 8,70 & 110,61 & 92,34 & 195,78 \\
\hline Heredia & 5,13 & 93,08 & 73,99 & 161,76 \\
\hline Colima & 6,46 & 96,09 & 76,83 & 167,97 \\
\hline Sabanilla & 6,78 & 101,96 & 83,05 & 178,46 \\
\hline
\end{tabular}

Fuente: (Quirós, 2009).

Cuadro 7. Análisis de potencias, para caso base, usando CPF.
Cuadro 8. Análisis de potencias, para caso base, usando OPF.

\begin{tabular}{|c|c|c|c|c|c|}
\hline Item & MW & MVAr & Item & MW & MVAr \\
\hline Generación & 1283,90 & 220,00 & Generación & 1291,00 & $-247,80$ \\
\hline Carga & 1262,70 & 369,00 & Carga & 1262,70 & 369,00 \\
\hline Perdidas & 21,30 & 347,90 & Perdidas & 21,20 & 252,10 \\
\hline
\end{tabular}


una disminución de la reserva de potencia reactiva. De lo anterior, se puede concluir que para las condiciones analizadas el sistema está muy cerca del colapso de tensión.

Es importante observar en el Cuadro 6 como en algunas barras el margen de potencia reactiva aumenta hasta en 100 MVAr utilizando el OPF, con respecto al caso en donde se hace uso del CPF. Para puntualizar lo anterior, se debe observar que en la barra de El Este el margen de potencia reactiva, considerando ambas contingencias, aumenta en 101 MVAr y 103 MVAr respectivamente.

\subsubsection{Demanda máxima de invierno, 18:30 horas}

Al igual que el caso anterior, para el caso base de "demanda máxima de invierno 2010", se realizó la simulación, tanto con la herramienta del CPF, como con el OPF. En el Cuadro 7 se resume la potencia de la generación, el consumo de la carga y las pérdidas para el caso del CPF, mientras que en el Cuadro 8 se resume los mismos datos utilizando la herramienta OPF

Al simular el caso de FNC igual a 567 con OPF, se obtiene una disminución del 0,47\%, para las pérdidas de potencia activa; mientras que para el caso de la potencia reactiva, ésta disminuye en un 27,54\%, respecto a la simulación con el CPF.

Para la topología considerada de la red para el año 2010, y de acuerdo al FNC estimado de $567 \mathrm{MW}$, no hay alguna contingencia que provoque la inestabilidad de la red (Quirós, 2009). Por esta razón, se realiza un redespacho de generación para observar las condiciones de FNC, que presenta la inestabilidad del sistema.

A partir del punto de colapso de tensión del sistema, se determina nuevamente que las contingencias más severas sobre la red de potencia son: la salida de la Barra A de Arenal (ARENAL) y la salida de la línea Cariblanco - San Miguel (CU1) respectivamente. Definidas las contingencias más severas, se procede a obtener las curvas $\mathrm{P}-\mathrm{V}$ y V-Q y observar efectivamente la cercanía al punto de colapso de tensión del sistema.

Se debe observar de la Figura 7 que al considerar la solución con OPF se determina que la tensión de colapso aumenta en aproximadamente 0,033 p.u.

Una vez obtenida la curva $\mathrm{P}-\mathrm{V}$, se puede observar que ambas contingencias tienen puntos de colapso diferente, por lo que se aclara que las curvas V-Q, considerando la salida de la línea Cariblanco - San Miguel, se realizan para un FNC de 842,17 MW. Por otro lado, para realizar las mismas curvas, pero considerando la salida de la Barra A de Arenal, se utiliza un FNC de 774,15 MW.

En las Figuras 8 y 9 se detallan las curvas V-Q para la barra de $230 \mathrm{kV}$ de La Caja, tanto con la herramienta del CPF como con la del OPF, para la salida de la línea Cariblanco - San Miguel y la salida de la barra A de Arenal, respectivamente.

En la Figura 8, se obtiene que el margen de potencia reactiva de la barra de $230 \mathrm{kV}$ en la Subestación La Caja, es de 7,11 MVAr ante la salida de la línea Cariblanco - San Miguel, mientras que para el mismo caso utilizando el OPF, el valor de 115,38 MVAr.

En el caso de la salida de la barra A de Arenal, la Figura 9 muestra que el margen de potencia reactiva en la barra de $230 \mathrm{kV}$ en la Subestación La Caja utilizando el CPF es de 107,48 MVAr y 220,32 MVAr utilizando el OPF.

En forma más específica, en el Cuadro 9 se observa como el margen de potencia reactiva en las barras del sistema aumenta significativamente utilizando el OPF. Tal como sucede para la barra de Alajuelita, cuando la tensión aumenta hasta en casi 0,0431 p.u (para la falla de la línea Cariblanco - San Miguel).

Una vez disponibles las curvas $\mathrm{P}-\mathrm{V}$ para el caso de demanda máxima de invierno, es posible realizar un resumen del margen de potencia reactiva, para cada barra del Cuadro 2. En el Cuadro 10, se muestra la mejoría en el margen de potencia reactiva de cada barra del sistema, siendo la salida de la barra A de Arenal, el caso que provoca la mayor inestabilidad del sistema. Esta situación se refleja en la cantidad de potencia activa, que se transfiere del norte hacia el centro de carga del país.

A partir del Cuadro 10, se detalla que ante las contingencias consideradas, la red experimenta una disminución de la reserva de potencia reactiva, que la acerca al colapso de tensión. De lo anterior, se puede concluir que para las 
La Caja 230

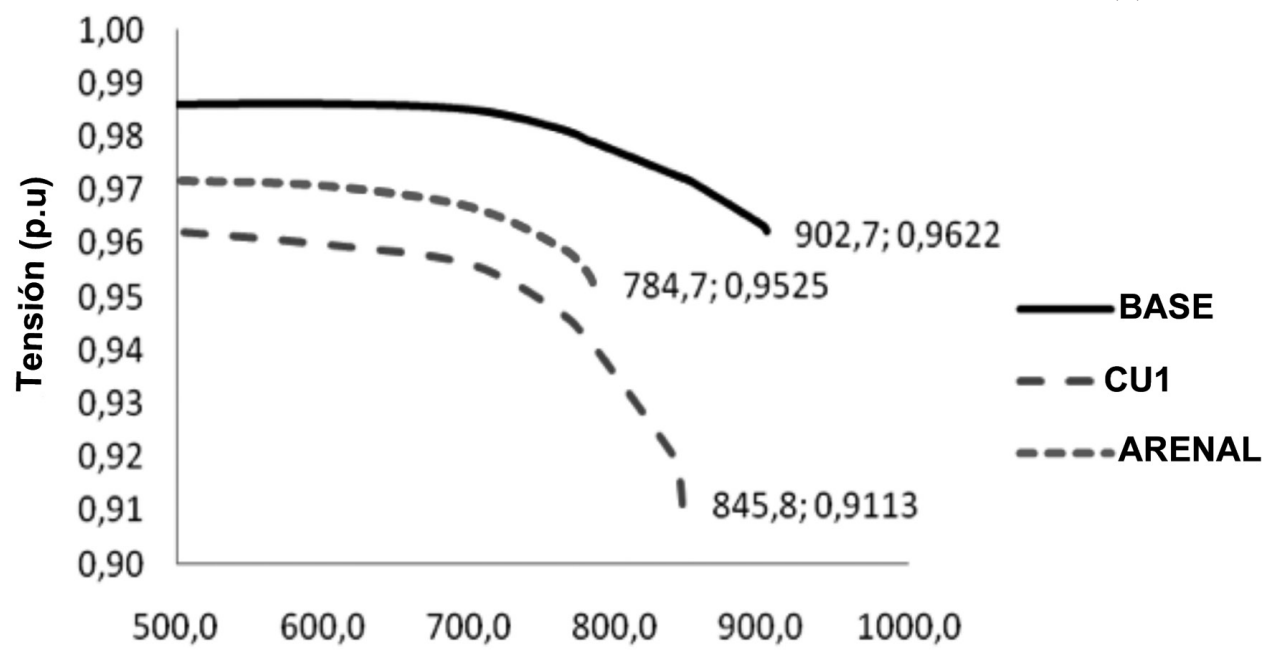

Flujo Norte - Centro (MW)

La Caja 230

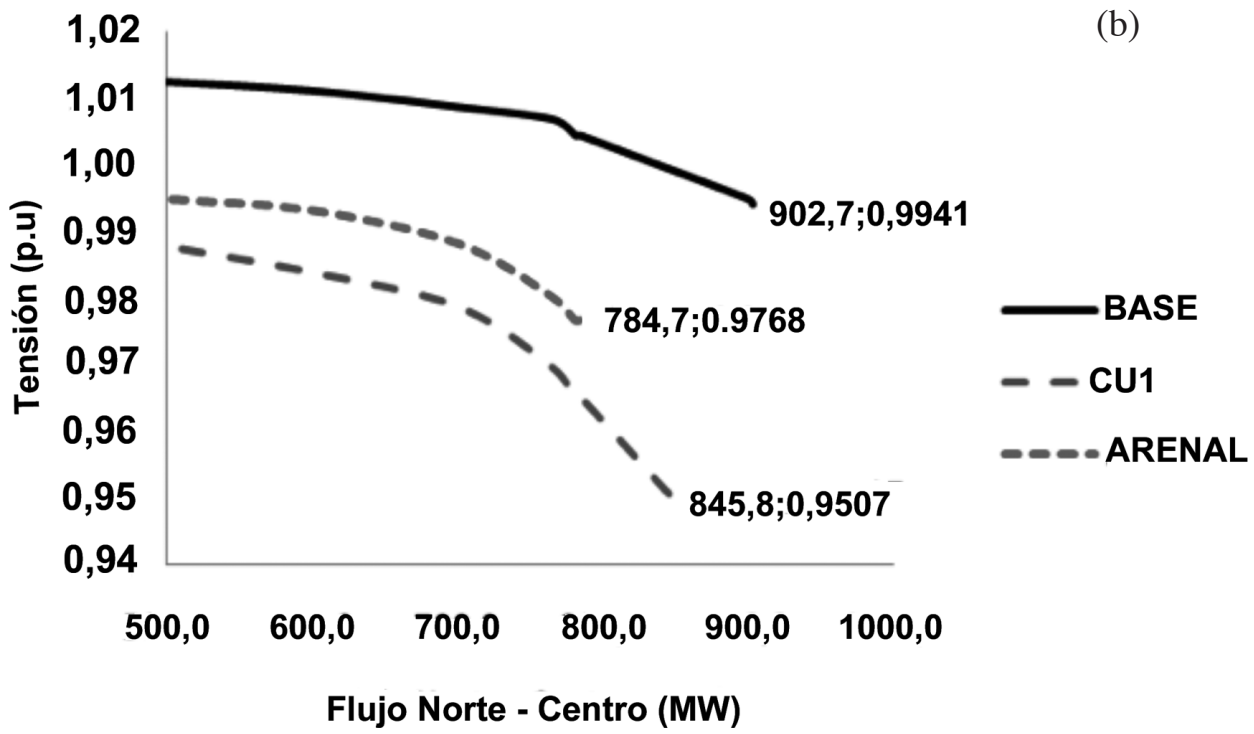

Figura 7. Curva P-V en La Caja 230 para caso base de demanda máxima de invierno, considerando el FNC (a) CPF (b) OPF. Fuente: (Quirós, 2009) 


\section{La Caja 230}

(a)

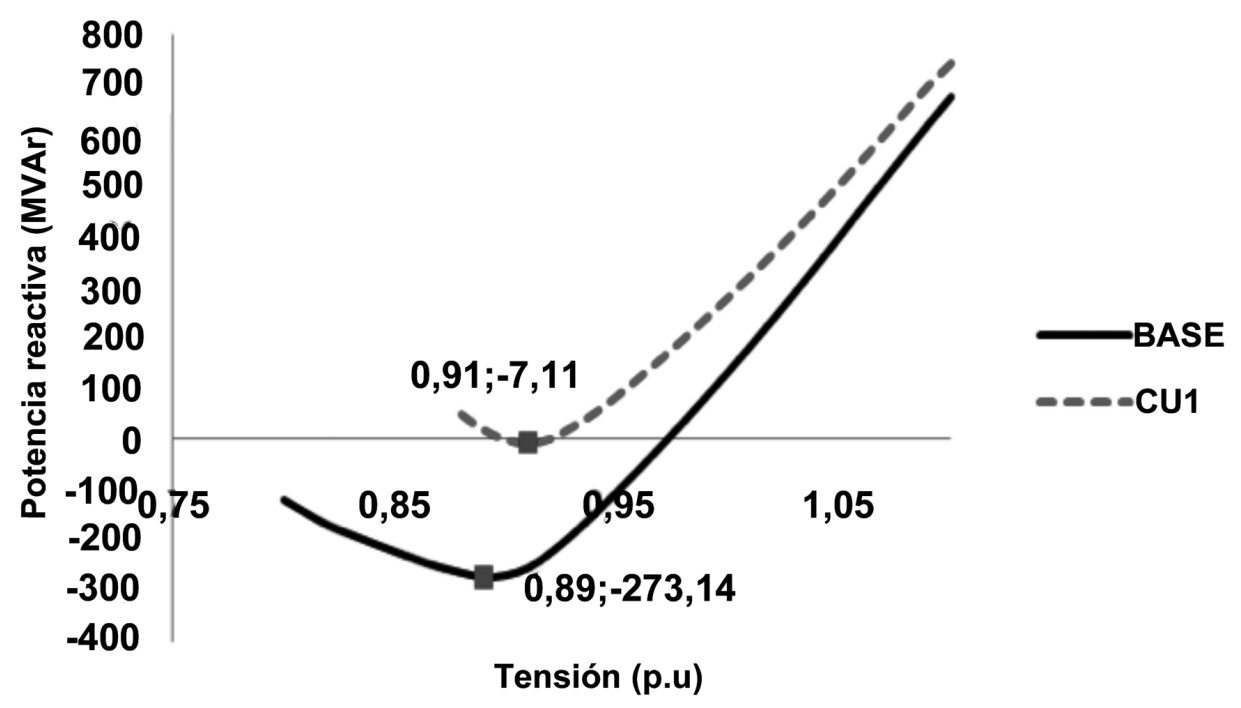

La Caja 230

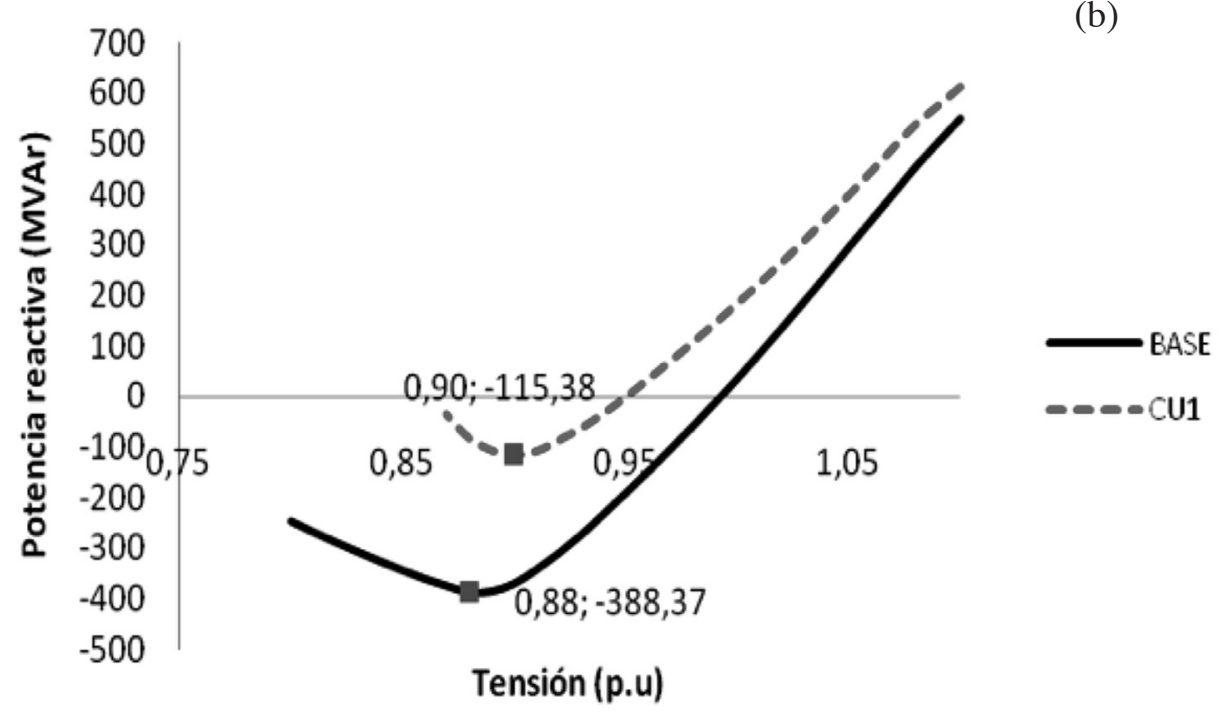

Figura 8. Curva V-Q en La Caja 230 para caso base de invierno máxima y la salida de la línea Cariblanco - San Miguel (a) CPF (b) OPF.

Fuente: (Quirós, 2009) 
La Caja 230

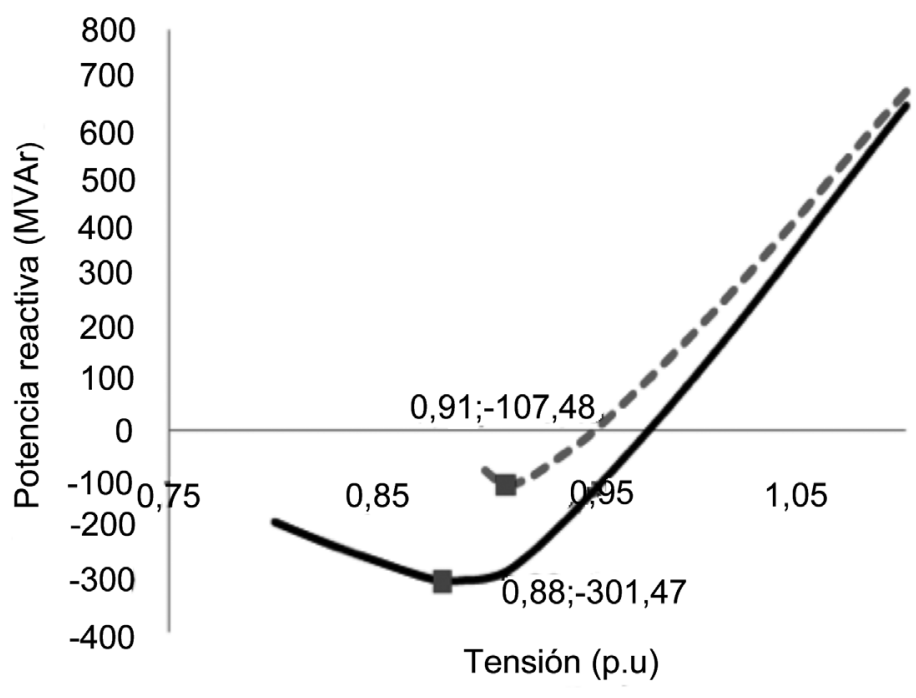

La Caja 230

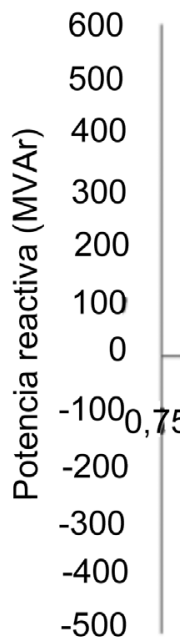

(a)

BASE

ARENAL

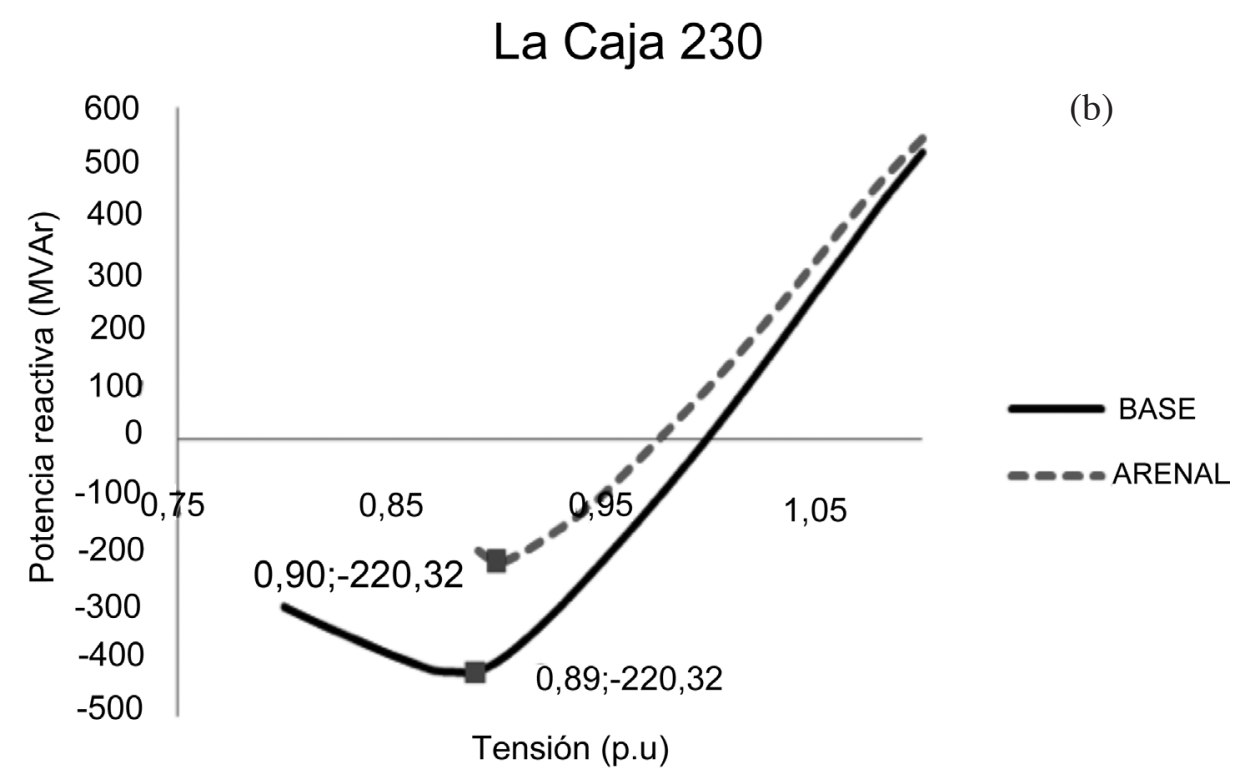

Figura 9. Curva V-Q en La Caja 230 para caso base de demanda máxima de invierno y la salida de la barra A de Arenal (a) CPF (b) OPF.

Fuente: (Quirós, 2009) 
Cuadro 9. Puntos de colapso del SEN, obtenidos de las curvas P-V, en demanda máxima de invierno.

\begin{tabular}{|c|c|c|c|c|c|c|c|c|}
\hline \multirow[t]{3}{*}{ Nombre de la barra } & \multicolumn{4}{|c|}{ Falla línea Cariblanco - San Miguel } & \multicolumn{4}{|c|}{ Falla barra A de Arenal } \\
\hline & \multicolumn{2}{|c|}{$\mathrm{CPF}$} & \multicolumn{2}{|c|}{ OPF } & \multicolumn{2}{|c|}{$\mathrm{CPF}$} & \multicolumn{2}{|c|}{ OPF } \\
\hline & $\mathrm{P}(\mathrm{MW})$ & V (p.u) & $\mathrm{P}(\mathrm{MW})$ & $V(p . u)$ & $\mathrm{P}(\mathrm{MW})$ & V (p.u) & $\mathrm{P}(\mathrm{MW})$ & $\mathrm{V}(\mathrm{p} . \mathrm{u})$ \\
\hline Barranca & 845,8 & 0,9326 & 845,8 & 0,9672 & 784,7 & 0,9622 & 784,7 & 0,9838 \\
\hline Garabito & 845,8 & 0,9361 & 845,8 & 0,9699 & 784,7 & 0,9640 & 784,7 & 0,9848 \\
\hline La Caja & 845,8 & 0,9113 & 845,8 & 0,9507 & 784,7 & 0,9525 & 784,7 & 0,9768 \\
\hline Lindora & 845,8 & 0,9128 & 845,8 & 0,9518 & 784,7 & 0,9541 & 784,7 & 0,9780 \\
\hline San Miguel & 845,8 & 0,9165 & 845,8 & 0,9550 & 784,7 & 0,9584 & 784,7 & 0,9810 \\
\hline Escazú & 845,8 & 0,9442 & 845,8 & 0,9855 & 784,7 & 0,9799 & 784,7 & 1,0071 \\
\hline Desamparados & 845,8 & 0,9444 & 845,8 & 0,9857 & 784,7 & 0,9782 & 784,7 & 1,0060 \\
\hline Alajuelita & 845,8 & 0,9443 & 845,8 & 0,9874 & 784,7 & 0,9793 & 784,7 & 1,0086 \\
\hline El Este & 845,8 & 0,9536 & 845,8 & 0,9914 & 784,7 & 0,9852 & 784,7 & 1,0102 \\
\hline Heredia & 845,8 & 0,9401 & 845,8 & 0,9817 & 784,7 & 0,9772 & 784,7 & 1,0044 \\
\hline Colima & 845,8 & 0,9432 & 845,8 & 0,9843 & 784,7 & 0,9809 & 784,7 & 1,0074 \\
\hline Sabanilla & 845,8 & 0,9457 & 845,8 & 0,9839 & 784,7 & 0,9828 & 784,7 & 1,0067 \\
\hline
\end{tabular}

Fuente: (Quirós, 2009).

condiciones analizadas, el sistema está muy cerca del colapso de tensión del sistema de potencia. Finalmente, es importante observar como en algunas barras el margen de potencia reactiva aumenta hasta en 100 MVAr utilizando el OPF, con respecto al caso en donde se hace uso del CPF. Como ejemplo puntual, puede observarse que en la barra de Escazú el margen de potencia reactiva aumenta en 114 MVAr ó 110 MVAr, según sea la contingencia.

\section{CONCLUSIONES}

Del análisis realizado se concluye que:

- Es posible obtener un incremento considerable en el margen de cargabilidad del sistema cuando el análisis se ejecuta utilizando la herramienta de flujos óptimos de potencia, en comparación con un flujo de potencia convencional.

- El uso de la herramienta OPF en el SEN debe ser analizada para un mejor manejo de los recursoseléctricosenlarednacional.Haciendo uso de los flujos de potencia óptimos se podrían obtener considerablemente mejores resultados, el punto de colapso del sistema puede ser aumentado y consecuentemente la probabilidad de colapsos de tensión se verán disminuidos.

- El punto de colapso de tensión, para la topología estimada del SEN para el año 2010 sin falla, se manifiesta cuando se tiene un Flujo de potencia Norte Centro de 924 MW 
para el escenario de demanda de verano. En el caso de invierno este valor es de $902 \mathrm{MW}$.

- La salida de la barra A de Arenal es la contingencia más severa del SEN, para la topología estimada de la red para el año 2010. Así mismo, la segunda contingencia más grave es la salida de la línea Cariblanco - San Miguel.

- Considerada la contingencia más crítica de la red, el punto de colapso de tensión del sistema disminuyó a 823 MW y 784 MW, para los escenarios de demanda de verano e invierno respectivamente.

- Se determinóque el margende potenciareactiva en las barras del SEN, para la contingencia más severa, es de aproximadamente $70 \mathrm{MVAr}$ (demanda máxima de verano). Mientras que para el escenario de demanda máxima de invierno este margen aumentó a 100 MVAr.

\section{NOMENCLATURA}

$\begin{array}{ll}B_{k m} & \begin{array}{l}\text { Parte imaginaria del elemento } \\ k m \text { en la matriz de admitancia }\end{array} \\ C E N C E & \begin{array}{l}\text { Centro Nacional de Control } \\ \text { de Energía }\end{array} \\ C P F & \begin{array}{l}\text { Flujo de potencia conven- } \\ \text { cional, CPF por sus siglas en } \\ \text { inglés }\end{array}\end{array}$

FNC Entiéndase Flujo de potencia Norte Centro

$G_{k m} \quad$ Parte real del elemento $\mathrm{km}$ en la matriz de admitancia

Cuadro 10. Margen de potencia reactiva, en demanda máxima de invierno.

\begin{tabular}{|c|c|c|c|c|}
\hline \multirow[t]{2}{*}{ Nombre de la barra } & \multicolumn{2}{|c|}{$\begin{array}{c}\text { Falla línea Cariblanco - San Miguel } \\
\text { Margen de Q (MVAr) }\end{array}$} & \multicolumn{2}{|c|}{$\begin{array}{l}\text { Falla barra A de Arena } \\
\text { Margen de Q (MVAr) }\end{array}$} \\
\hline & $\mathrm{CPF}$ & OPF & $\mathrm{CPF}$ & OPF \\
\hline Barranca & 7,67 & 103,33 & 95,76 & 173,81 \\
\hline Garabito & 3,89 & 102,15 & 89,85 & 171,41 \\
\hline La Caja 230 & 7,11 & 115,38 & 107,48 & 220,32 \\
\hline La Caja 138 & 5,65 & 119,42 & 106,74 & 221,45 \\
\hline Lindora & 6,61 & 115,29 & 101,20 & 221,83 \\
\hline San Miguel 230 & 4,02 & 121,25 & 110,88 & 225,82 \\
\hline San Migue 138 & 5,47 & 122,95 & 108,46 & 223,44 \\
\hline Escazú & 6,45 & 120,33 & 104,53 & 214,11 \\
\hline Desamparados & 8,21 & 120,69 & 100,02 & 202,17 \\
\hline Alajuelita & 7,99 & 117,91 & 102,19 & 205,70 \\
\hline El Este & 8,74 & 124,36 & 103,76 & 210,34 \\
\hline Heredia & 6,40 & 120,17 & 102,76 & 214,80 \\
\hline Colima & 6,92 & 124,37 & 104,39 & 219,95 \\
\hline Sabanilla & 6,12 & 122,20 & 104,47 & 212,22 \\
\hline
\end{tabular}

Fuente: (Quirós, 2009). 

$\tilde{I} \quad$ Fasor de corriente fluyendo en la barra $i$
ICE Instituto Costarricense de Electricidad

OPF Flujo óptimo de potencia, OPF por sus siglas en inglés

$\begin{array}{ll}P & \text { Potencia activa } \\ p . c & \text { Entiéndase punto crítico } \\ p . o & \text { Entiéndase punto óptimo } \\ p . u & \text { Por unidad }\end{array}$

PSS/E Simulador de Sistemas de Potencia para Ingeniería, PSS/E por sus siglas en inglés.

$Q \quad$ Potencia reactiva

SEN Sistema Eléctrico Nacional

UEN Unid. Estratégica de Negocios

$\tilde{V}_{i}$

V

VA Volt-Ampere, unidad de medida de potencia aparente

$V A r \quad$ Volt-Ampere reactivo, unidad de medida de potencia reactiva

W Watt, unidad de medida de potencia activa

$Y_{i i} \quad$ Admitancia propia de la barra $i$

$Y_{i j} \quad$ Admitancia mutua entre la barra $i$ y $j$

$\Theta_{k m} \quad$ Ángulo del número complejo $\mathrm{km}$ en la matriz de admitancia

\section{REFERENCIAS BIBLIOGRÁFICAS}

CIGRE: Task Force 38.02.10. (1992). Modelling of voltage collapse including dynamic phenomena.

ICE: Centro Nacional de Control de Energía. (2001). Informe del apagón nacional del 6 de marzo del Año 2001. San José, Costa Rica.

Kundur, P. (1994). Power System Stability and Control. McGrawHill, Inc.

Quirós, J. (2009). Incremento del margen de estabilidad de tensión del Sistema Eléctrico Nacional. Tesis de Licenciatura. Escuela de Ingeniería Eléctrica. Universidad de Costa Rica: San José.

Taylor, C. (1994). Power System Voltage Stability. McGraw-Hill Publishers.

Tiranuchit, A., Ewerbring, L. M., Duryea, R. A., Thomas, R. J., \& Luk, F. T. (1988). Towards a computationally feasible on-line voltage instability index. IEEE Transaction on Power Systems, 3(2), 669-675.

Van Cutsem, T., \& Vournas, C. (1998). Voltage Stability of Electric Power Systems. Kluwer Academic Publishers.

Verayiah, R., \& Zainal, I. Z. (2008). A study on static voltage collapse proximity indicators. $2^{\text {nd }}$ IEEE International Conference on Power and Energy (PECon 08), Johor Bahru. 1-3 December, 531-536.

\section{SOBRE LOS AUTORES}

\section{Jairo Quirós Tortós}

Profesor Interino. Departamento de Máquinas Eléctricas y Sistemas de Potencia, Escuela de Ingeniería Eléctrica, Universidad de Costa Rica. Su área de interés, son los sistemas de protección en alta tensión.E-mail: jairoquirostortos@ieee.org

\section{Eddie A. Araya Padilla}

Profesor Catedrático. Departamento de Máquinas Eléctricas y Sistemas de Potencia, Escuela de Ingeniería Eléctrica, Universidad de Costa Rica. Sus áreas de interés son la dinámica no lineal, los dispositivos FACTS y la generación eólica.E-mail: earaya@eie.ucr.ac.cr 